\title{
Lentivirus vector-mediated genetic manipulation of oncogenic pathways induces tumor formation in rabbit brain
}

\author{
FARIZAN AHMAD ${ }^{1,2 *}$, ANNA HYVÄRINEN ${ }^{1,3-5^{*}}$, AGNIESZKA PIRINEN $^{1}$, VENLA OLSSON $^{1}$ \\ JAANA RUMMUKAINEN $^{6}$, ARTO IMMONEN ${ }^{7}$, JOHANNA NÄRVÄINEN ${ }^{1,8}$, PASI TUUNANEN $^{1,9}$, \\ TIMO LIIMATAINEN $^{1,10,11}$, VIRVE KÄRKKÄINEN ${ }^{7}$, JARI KOISTINAHO ${ }^{1,12}$ and SEPPO YLÄ-HERTTUALA ${ }^{1}$ \\ ${ }^{1}$ A.I Virtanen Institute for Molecular Sciences, University of Eastern Finland, FI-70211 Kuopio, Finland; \\ ${ }^{2}$ Department of Neurosciences, School of Medical Sciences, Universiti Sains Malaysia, Kubang Kerian, \\ Kelantan 16150, Malaysia; ${ }^{3}$ Department of Pediatric Surgery, Tampere University Hospital, FI-33520 Tampere; \\ ${ }^{4}$ Tampere University, FI-33014 Tampere; ${ }^{5}$ Department of Surgery, North Karelia Central Hospital, \\ FI-80210 Joensuu; ${ }^{6}$ Department of Pathology, Kuopio University Hospital; ${ }^{7}$ Department of Neurosurgery, \\ Neurocenter in Kuopio University Hospital, FI-70029 Kuopio; ${ }^{8}$ VTT Technical Research Centre of Finland, \\ FI-70210 Kuopio; ${ }^{9}$ Department of Clinical Neurophysiology, Kuopio University Hospital, FI-70029 Kuopio; \\ ${ }^{10}$ Research Unit for Medical Imaging, Physics and Technology, University of Oulu, FI-90014 Oulu; \\ ${ }^{11}$ Department of Diagnostic Radiology, Oulu University Hospital, FI-90220 Oulu; ${ }^{12}$ Neuroscience Center, \\ Helsinki Institute of Life Sciences, University of Helsinki, FI-00014 Helsinki, Finland
}

Received March 16, 2020; Accepted December 4, 2020

DOI: $10.3892 / \mathrm{mmr} .2021 .12061$

\begin{abstract}
Translation of promising experimental therapies from rodent models to clinical success has been complicated as the novel therapies often fail in clinical trials. Existing rodent glioma models generally do not allow for preclinical evaluation of the efficiency of novel therapies in combination with surgical resection. Therefore, the aim of the present study was to develop a larger animal model utilizing lentivirus vector-mediated oncogenic transformation in the rabbit brain. Lentiviruses carrying constitutively active AKT and H-Ras oncogenes, and p53 small interfering (si)RNA were introduced into newborn rabbit neural stem cells (NSCs) and intracranially implanted into rabbits' brains to initiate tumor formation. In one of the ten rabbits a tumor was detected 48 days after the implantation of transduced NSCs. Histological features of the tumor mimic was similar to a benign Grade II ganglioglioma. Immunostaining demonstrated that the tissues were positive for AKT and H-Ras. Strong expression of GFAP and Ki-67 was also detected. Additionally, p53 expression was notably lower in the tumor area. The implantation of AKT, H-Ras and
\end{abstract}

Correspondence to: Professor Seppo Ylä-Herttuala, A.I Virtanen Institute for Molecular Sciences, University of Eastern Finland, Neulaniementie 2, Bioteknia, FI-70211 Kuopio, Finland

E-mail: seppo.ylaherttuala@uef.fi

*Contributed equally

Key words: ganglioglioma, rabbit brain tumor model, oncogenes, lentivirus vector p53 siRNA transduced NSCs for tumor induction resulted in ganglioglioma formation. Despite the low frequency of tumor formation, this preliminary data provided a proof of principle that lentivirus vectors carrying oncogenes can be used for the generation of brain tumors in rabbits. Moreover, these results offer noteworthy insights into the pathogenesis of a rare brain tumor, ganglioglioma.

\section{Introduction}

Gliomas are the most common type of primary brain tumors in adults and represent $>50 \%$ of all primary brain tumors. In adults, glioblastoma is the most common histological type (1). It is characterized by aggressive growth, rapid progression of the disease and poor prognosis. In children, high-grade gliomas represent only a minority of primary brain tumors, while the low-grade tumors predominate (2). Compared with adults, pediatric tumor types are generally more sensitive to irradiation and chemotherapy (3). In all age groups current treatments for high-grade gliomas (surgery, radiotherapy and chemotherapy) very rarely yield long-term control of the disease.

Several rodent glioma models have been established and utilized for malignant glioma research. These models generally involve implantation of cultured human or rodent glioma cells into the brains of nude mice or syngeneic mice or rats, respectively $(4,5)$. Previously, spontaneously arising tumors or tumors arising after exposure of animals to carcinogenic chemicals, such as N-ethylnitrosourea, were used for the establishment of malignant glioma cell lines (6-9). More recently, development of gene transfer techniques has provided novel tools for creating cancer models with desired genetic profiles. Lentivirus vectors are useful tools for stable long-term 
expression of transgenes. They have been utilized for oncogene transfer to induce malignant transformation and to study the role of different oncogenes in malignant transformation $(10,11)$. Akt, H-Ras and p53 are all integral components of signaling pathways (PI3K-Akt-mTOR and EGFR-Ras-MEK/ERK pathways) or cell cycle regulation (p53). Due to the central roles of these genes in glioblastoma pathogenesis they were selected for transformation in the present study (12).

Translation of promising experimental therapies from rodent models to clinical success has been complicated as the novel therapies often fail in clinical trials. Rodent glioma models generally do not allow for preclinical evaluation of the efficiency of novel therapies in combination with surgical resection, which is an essential part of current therapeutic protocols (13-15). Utilizing a larger laboratory animal model, such as rabbit, enables the testing of novel treatments and local administration techniques in combination with surgery. For example, preclinical evaluation of endovascular therapies require larger animal models, such as rabbit (16). However, no rabbit glioma cell lines exist. The aim of the present study was to investigate the utility of lentivirus vector-mediated oncogenic transformation for establishing rabbit glioma tumors and to create a novel rabbit model for malignant glioma. In the present study, a malignant glioma rabbit model was not created. However, the study produced unexpected, but noteworthy findings deciphering the pathogenesis of a rare benign brain tumor, ganglioglioma.

\section{Materials and methods}

Animals. The experimental design is presented in the Fig. 1. New Zealand White (NZW, Harlan Sprague Dawley, Inc.) rabbits were used for this study. For implantation of the transduced neural stem cells (NSCs), female NZW rabbits (age, 5.5 months) that weighed between $2.5-3.5 \mathrm{~kg}$ were used. The study was carried out according to Finnish National and European Union legislation and guidelines. The permission for animal studies was acquired from the Project Authorization Board of the Regional State Administrative Agency. All animals were maintained at the Lab Animal Center of the University of Eastern Finland (Kuopio, Finland). Housing temperatures for mice and rabbits were $22^{\circ}$ and $18^{\circ} \mathrm{C}$, respectively. Moreover, animals were housed under a normal atmosphere with $55 \pm 15 \%$ relative humidity and $12 \mathrm{~h}$ light-dark cycle. The animals had free access to food and water. Animal welfare was monitored twice a day, once a day by the research group members and once a day by the personnel of the lab animal center. MRI imaging was carried out in the Nuclear Magnetic Resonance Imaging Laboratory at the A. I. Virtanen Institute for Molecular Sciences, University of Eastern Finland, with facilities suitable for experimental animal imaging. Excel (version 2011, Microsoft Corporation) was used to create the graph. For anesthesia, Domitor ${ }^{\circledR}$ (medetomidine $1 \mathrm{mg} / \mathrm{ml}$; Orion Corporation) $0.25-0.5 \mathrm{mg} / \mathrm{kg}$ subcutaneous (s.c.) and $\operatorname{Ketalar}^{\circledR}$ (ketamine $50 \mathrm{mg} / \mathrm{ml}$; Pfizer, Inc.) $15-25 \mathrm{mg} / \mathrm{kg}$ s.c. for rabbits, and $75 \mathrm{mg} / \mathrm{kg}$ ketamine and $1.0 \mathrm{mg} / \mathrm{kg}$ medetomidine for s.c. mice. Before use, $1 \mathrm{ml}$ Hypnorm (containing $0.315 \mathrm{mg}$ fentanyl $/ \mathrm{ml}$ and $10 \mathrm{mg}$ fluanisone/ml) was mixed with $1 \mathrm{ml}$ Dormicum (containing $5 \mathrm{mg}$ midazolam) and $2 \mathrm{ml}$ sterile water and the solution was

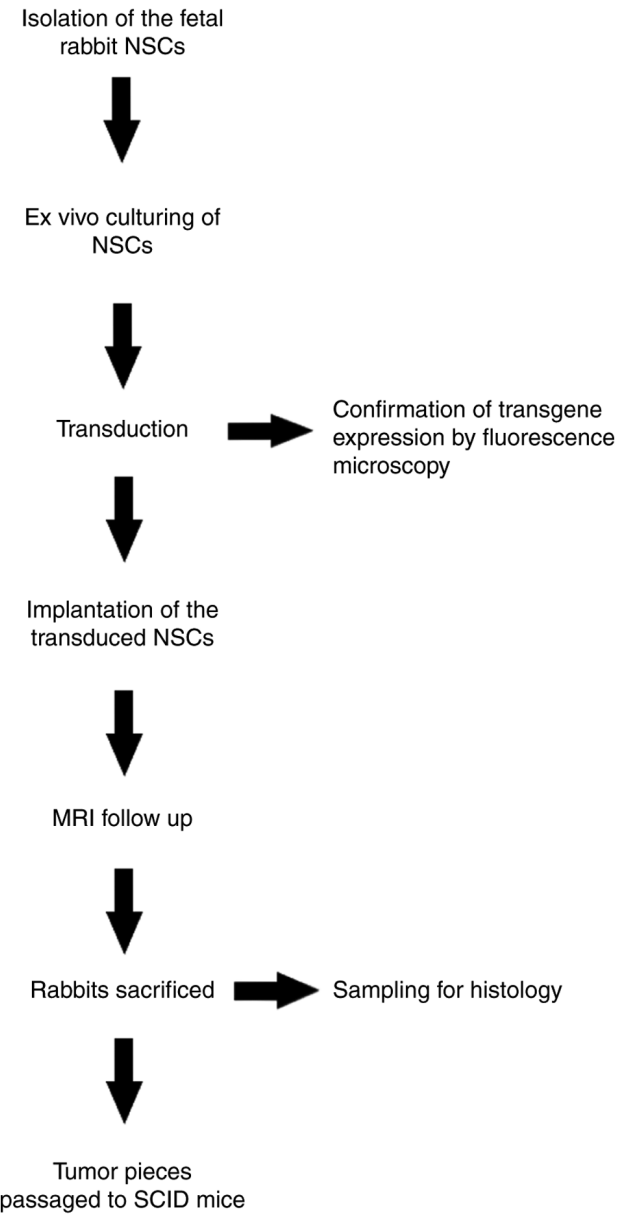

Figure 1. Experimental protocol. Isolation of the fetal NSCs was followed by short ex vivo culturing to expand the cell population and allow the transduction with the Tomo-H-Ras and Tomo-AKT lentiviral vectors (MOI 3) together with the Adeno-cre vector (MOI 30) needed for activation of $\mathrm{H}-\mathrm{R}$ as and AKT transgene expression. The next day, the transduced cells were implanted into the brains of 10 rabbits. Part of the transduced cells was plated for verification of transgene expression with fluorescence microscopy 7 days after transduction. In addition to sampling for histology, small pieces of the resulting ganglioglioma tumor were passaged into severe combined immunodeficient mice. NSCs, neural stem cells.

used for anesthesia of the mice at a dose of $0.1 \mathrm{ml} / 10 \mathrm{~g}$, intraperitoneal $(0.7875 \mathrm{mg} / \mathrm{kg}$ fentanyl, $25 \mathrm{mg} / \mathrm{kg}$ fluanison and $12.5 \mathrm{mg} / \mathrm{kg}$ midazolam) (17). Duration of the experiment was 141 days and at the end rabbits were sacrificed by decapitation under deep overdosing terminal anesthesia. One rabbit died under anesthesia during MRI imaging at day 141. The exact reason for the death of this animal remains unknown since the animal was asymptomatic before anesthesia induction. The criteria for euthanasia included neurological deterioration or poor physical condition. No control rabbits receiving Mock transduced NSCs were used to avoid unnecessary use of animals and no statistical analyses were performed. The severe combined immunodeficient (SCID) mice (Harlan Sprague Dawley, Inc.) were sacrificed with $\mathrm{CO}_{2}$ (gas flow $41 \%$ of the chamber volume/min) and death was confirmed by cervical dislocation. For this experiment, two male (age, 33 weeks; weight, $30 \mathrm{~g}$ ) mice were used.

Cell cultures. NSCs were generated from the hippocampus and lateral ventricle wall of the newborn rabbit pups as 


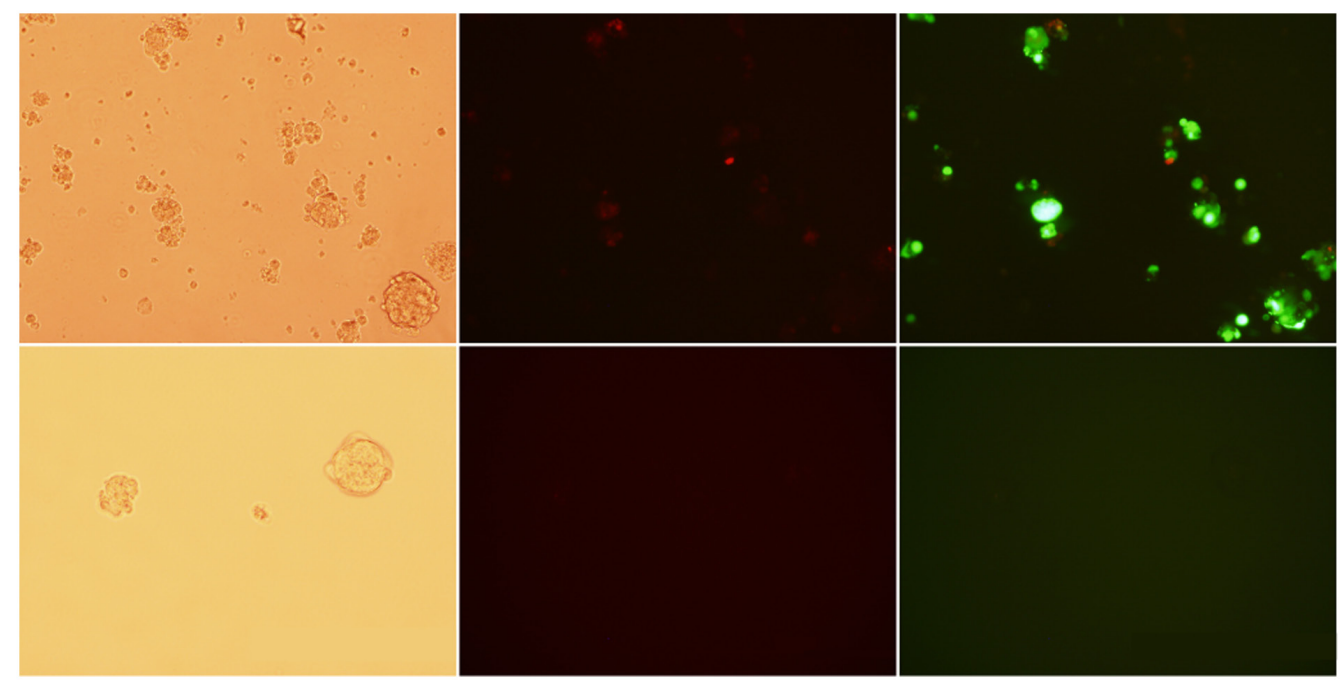

Figure 2. Transduction of rabbit NSCs with Cre/LoxP controlled pTomo-AKT, pTomo-H-Ras and Adeno-cre activation. NSCs were imaged 7 days after transduction and showed activation of AKT and H-Ras by Adeno-cre. Bright field images (left), as well as red (middle) and green (right) fluorescence images indicating AKT and H-Ras expression, respectively, are shown. Images in the upper row represent transduced cells and in the lower row show untransduced control stem cells from a different batch. Fluorescence microscopy showed EGFP expression in 38\% and co-expression of EGFP and RFP expression in 6\% of the transduced NSCs. The EGFP-expressing cells that did not co-express RFP (32\%) represent the cells that were successfully transduced with Adeno-Cre vector for activation of Akt and/or H-Ras transgene expression. All images were taken at x10 magnification. NSCs, neural stem cells; EGFP, enhanced green fluorescent protein; RFP, red fluorescent protein.

described by Clarke et al (18) and Supporting Materials and Methods from Castrén et al (19) and plated in culture medium containing $2 \mathrm{mM}$ L-glutamine, $15 \mathrm{mM}$ Hepes, $100 \mathrm{U} / \mathrm{ml}$ penicillin, $100 \mathrm{mg} / \mathrm{ml}$ streptomycin, B27 supplement, $20 \mathrm{ng} / \mathrm{ml}$ epideral growth factor (EGF; Thermo Fisher Scientific, Inc.), and $10 \mathrm{ng} / \mathrm{ml}$ fibroblast growth factor (FGF)-2 (PeproTech EC Ltd.) in DMEM/F12 medium (Thermo Fisher Scientific, Inc.). EGF (20 ng/ml) and FGF-2 (10 ng/ml) were added every 3rd day, and half of the medium was replaced with new medium every 3-4 day. The cells were incubated at $+37^{\circ} \mathrm{C}$. The stem cell isolation procedure was repeated twice. Plasmid and viral vectors for AKT and H-Ras were generated as previously described $(11,20)$. Briefly, the pTomo H-RasV12 and pTomo HA-AKT Cre-loxP-controlled lentiviral vectors were constructed containing a human cytomegalovirus immediate-early promoter (CMV)-loxP-red fluorescent protein (RFP)-loxP-Flag-H-RasV12/HA-RAS followed by internal ribosomal entry site (IRES)-green fluorescent protein (GFP). The stuffer fragment, RFP, keeps the translation of Flag H-RasV12 in an 'off' state. The excision of the stuffer sequence by Cre recombinase results in the expression of Flag H-RasV12 or HA-AKT $(11,20)$. Constitutively active AKT and H-Ras oncogenes were introduced into NSCs using lentiviral vectors (provided by Dr Inder Verma, Salk Institute, La Jolla, California, USA) at MOI 3. The same lentivirus vector without a transgene (Tomo-MOCK) was used for transduction of the negative control cells. Additionally, NSCs were also transfected with lentivirus vectors carrying mouse small interfering (si)RNA p53 (5'-CTGTCTAGACAAAAA ACAAGTACATGTGTAATAGCTCCTCGATGTCTCTTG AACATGCAGGAGCTATTACACATGTACTTGTGGGAT CTGTGGGTCTCATACA-3') constructed using CMV-IE promoter in pLV1-hPGK-GFP-WPRE plasmid (provided by Dr Inder Verma, Salk Institute) (21) at MOI 30. Adeno-Cre was used for the activation of those vectors at MOI 30 (22).
For the transductions, the neurospheres were trypsinized to yield a single cell suspension to improve the exposure of each individual cell to the transducing vectors. For demonstration of the transgene expression in vitro, the cells were cultured for 7 days in the EGF- and FGF-containing medium to allow the transgene expression to start before fluorescence microscopy. However, for subcortical in vivo implantation, the NSCs were used 1 day after transduction. All transductions were carried out at the same time and cultured overnight at $37^{\circ} \mathrm{C}$ before being implanted into animals the next day (Fig. 2). The proportions of fluorescent marker gene expressing cells were detected with bright field and fluorescence microscopy with x10 magnification after the transductions.

Stem cell implantation. A total of 10 adult female rabbits were anesthetized by s.c. injections of $0.25-0.5 \mathrm{mg} / \mathrm{kg}$ ketamine and $15-25 \mathrm{mg} / \mathrm{kg}$ medetomidine. Anesthesia was maintained with 0.02-0.05 mg intravenous boluses of ketamine and medetomidine, respectively, via cannulated marginal ear vein (23). After shaving and cleaning the heads of the rabbits, a $3-\mathrm{cm}$ incision was made along the midline and the periosteum was opened, before a small 3-4 mm craniotomy was made using a dentist drill. A total of $3.0 \times 10^{4}$ viable cells suspended in $15 \mu \mathrm{l}$ Opti-MEM (Invitrogen; Thermo Fisher Scientific, Inc.) were then injected via a stereotactic injection at $5 \mathrm{~mm}$ caudally and $6 \mathrm{~mm}$ right from the sagittal suture into $2 \mathrm{~mm}$ depth from the dura surface. For the stereotactic injections, a stereotactic frame with a custom-made rabbit head holder and a Hamilton syringe were used. After the injection was made, the needle was slowly drawn out to prevent backflow of the injected cell suspension. The scalp was then sutured and all rabbits received $4 \mathrm{mg} / \mathrm{kg}$ Rimadyl ${ }^{\circledR}$ (carprofen; Pfizer, Inc.) s.c. For infection prophylaxis, all rabbits received $125 \mathrm{mg}$ kefuroxime (GlaxoSmithKline) s.c. during the operation and on the first postoperative day. 
Magnetic resonance imaging (MRI). MRI scanning was performed every week or 2 weeks to monitor tumor growth. Anesthesia for MRI scanning was carried out as aforementioned. MRI data was acquired using a horizontal 4.7 T magnet (Magnex Scientific; Varian, Inc.) interfaced to a Varian UNITY INOVA $^{\mathrm{TM}}$ console (Varian Medical Systems, Inc.) using an in-lab built surface RF coil in transmit/receive mode with loop diameter $2.5 \mathrm{~cm}$. T2-weighted images were measured using a spin echo sequence (echo time, $40 \mathrm{msec}$; repetition time, $2 \mathrm{sec}$ ). A total of 11 slices, each of $1.5-\mathrm{mm}$ thickness, were imaged. The matrix size was $128 \times 128$, with field of view $50 \mathrm{~mm}^{2}$ yielding an in-plane resolution of $390 \mu \mathrm{m}$. Total tumor volume was processed and analyzed using MATLAB ${ }^{\circledR}$ version 7.04 software (The MathWorks, Inc.).

Histology. Immediately after euthanasia, the animals were perfused with $1 \%$ paraformaldehyde $\mathrm{pH} 7.4$. Brains were immersion-fixed in $4 \%$ paraformaldehyde/0.15 $\mathrm{M}$ sodium phosphate buffer ( $\mathrm{pH} 7.4$ ) for $48 \mathrm{~h}$ at $4^{\circ} \mathrm{C}$. After fixation, the samples were dehydrated, embedded into paraffin, sectioned into $4 \mu \mathrm{m}$ sections and stained for light microscopy (x4, x10, $\mathrm{x} 20$ and $\mathrm{x} 40$ magnifications) with standard hematoxylin and eosin (H\&E) staining, including staining with hematoxylin (Hematoxylin solution according to Delafield; Sigma-Aldrich; Merck KGaA; cat. no. 03971; $250 \mathrm{ml}$ ) for $10 \mathrm{~min}$ and eosin (Eosin Y solution alcoholic; Sigma-Aldrich; Merck KGaA; cat. no. HT110132-1 L) for $30 \mathrm{sec}$, at room temperature.

Before the addition of the primary antibody, the sections were treated with normal serum (Vector Laboratories, Inc.) diluted 1:20 in PBS. After exposure to the primary antibody overnight at $4^{\circ} \mathrm{C}$ the sections were washed with PBS twice for 2 min each time and then exposed to the secondary antibody (Vectastain IgG horse anti-mouse antibody, Vector Laboratories, Inc.; 1:200) for $30 \mathrm{~min}$ at room temperature. The staining was carried out with Vectastain ABC Method based on avidin-biotin-HRP reaction (Vector Laboratories, Inc.) with DAB substrate. For counterstaining, Harris-hematoxyline was used. After counterstaining, the sections were dehydrated with EtOH dilutions with rising concentrations (50, 70, 95, 100 and 100\%; 3 min each), followed by incubation with xylene two times for $5 \mathrm{~min}$ at room temperature.

Primary antibodies used for immunostainings included mouse-anti-HA tag monoclonal antibody to detect H-Ras (1:50; monoclonal HA11; clone no. 16B12, Nordic BioSite), anti-flag M2 monoclonal antibody to detect AKT (1:2,000; cat. no. F1804, Sigma-Aldrich; Merck KGaA), CD31 mouse anti-human monoclonal antibody (1:50; Dako; Agilent Technologies, Inc.; cat. no. M0823), Ki-67 mouse anti-human monoclonal antibody (1:100; Dako; Agilent Technologies, Inc.; cat. no. M7240), GFAP mouse monoclonal antibody (1:250; Abcam; cat. no. ab4648) and p53 mouse monoclonal antibody (1:100; Santa Cruz Biotechnology, Inc.; cat. no. SC-55476). Antibody diluent with background (Dako; Agilent Technologies, Inc.; cat. no. S3022) was used for dilution of the primary antibodies and for negative control staining. Incubation with primary antibodies was carried out overnight at $4^{\circ} \mathrm{C}$. VECTASTAIN ${ }^{\circledR}$ Elite ABC Kit (Vector Laboratories, Inc.) was then used for detection.

Double staining for detecting H-Ras and AKT localization/co-localization was performed using mouse-anti-HA tag and anti-FLAG ${ }^{\circledR}$ M2 monoclonal antibodies. Briefly, for the first sequence, slides were incubated with primary antibody anti-flag tag overnight at $4^{\circ} \mathrm{C}$, biotinylated secondary antibody (Vectastain IgG horse anti-mouse antibody; Vector Laboratories, Inc.; cat. no. BA-2000-1.5) 1:200 for $30 \mathrm{~min}$ at room temperature, Avidin-Biotin-HRP (Vector Laboratories, Inc.) for $30 \mathrm{~min}$ and Peroxidase Substrate Solution (DAB; Invitrogen; Thermo Fisher Scientific, Inc.) for 1-5 min under microscopic observation. For the second staining, slides were incubated with mouse-anti-HA tag overnight at $4{ }^{\circ} \mathrm{C}$, biotinylated secondary antibody (horse anti-mouse) for $30 \mathrm{~min}$, Avidin-Biotin-AP (Vector Laboratories, Inc.) for $30 \mathrm{~min}$ and Alkaline Phosphatase Solution (Vector Blue) (Vector Laboratories, Inc.) for 5-30 min under microscopic observation. No nuclear counterstain was used in order to avoid difficulties in interpreting three-color tissue preparations. After staining, sections were air dried and mounted in aqueous medium (VWR International, LLC). Single-positive cells were stained brown for AKT, blue for H-Ras and dark blue-brown for colocalization of both.

Periodic acid-Schiff (PAS) and CD31 dual staining was performed to validate the existence of vasculogenic mimicry or vascular co-option in the tumor tissue. Slides were initially stained with general protocols for CD31 mouse anti-human monoclonal antibody. Following incubation with DAB, slides were immersed in PAS reagents for $5 \mathrm{~min}$, rinsed in distilled water three times for 1 min each, immersed in Schiff's reagents for $15 \mathrm{~min}$ at room temperature, washed under running tap water for $5 \mathrm{~min}$, and finally counterstained with hematoxylin solution for $90 \mathrm{sec}$ at room temperature. Slides were dehydrated under a series of alcohol concentrations and xylene, and were then mounted with Permount ${ }^{\mathrm{TM}}$ Mounting Medium. PAS reagents were purchased from Sigma-Aldrich (Merck KGaA).

Re-passaging of the tumor s.c. into SCID mice. To determine the reproducibility of the tumor, a small piece of the tumor was excised via an operation after day 141, cut into several pieces and s.c. implanted into immunodeficient SCID mice. SCID mice were anesthetized by s.c. injections of $75 \mathrm{mg} / \mathrm{kg}$ ketamine and $1.0 \mathrm{mg} / \mathrm{kg}$ medetomidine. After cleaning, a small incision was made on the back of the mice using a surgical scalpel. The tumor pieces were then inserted under the skin, one tumor piece/mouse, further from the midline incision. The incision was then sutured and the mice received $4 \mathrm{mg} / \mathrm{kg}$ carprofen s.c. For infection prophylaxis, the mice received $125 \mathrm{mg}$ kefuroxime s.c. during operation and on the first postoperative day. For histological analysis (see above), tumor growth was monitored until the proper size was reached, and then samples were taken 107 days after tumor implantation.

\section{Results}

Transductions of the NSCs. Fluorescence microscopy showed EGFP expression in 38\% and co-expression of EGFP and RFP in $\sim 6 \%$ of the transduced NCSs. Expression of RFP without simultaneous EGFP expression was not detected.

MRI and tumor growth. MRI data showed tumor growth after 48 days of NSC implantation (Fig. 3) in one of the ten rabbits (10\%). MRI follow-up showed slow growth of the 


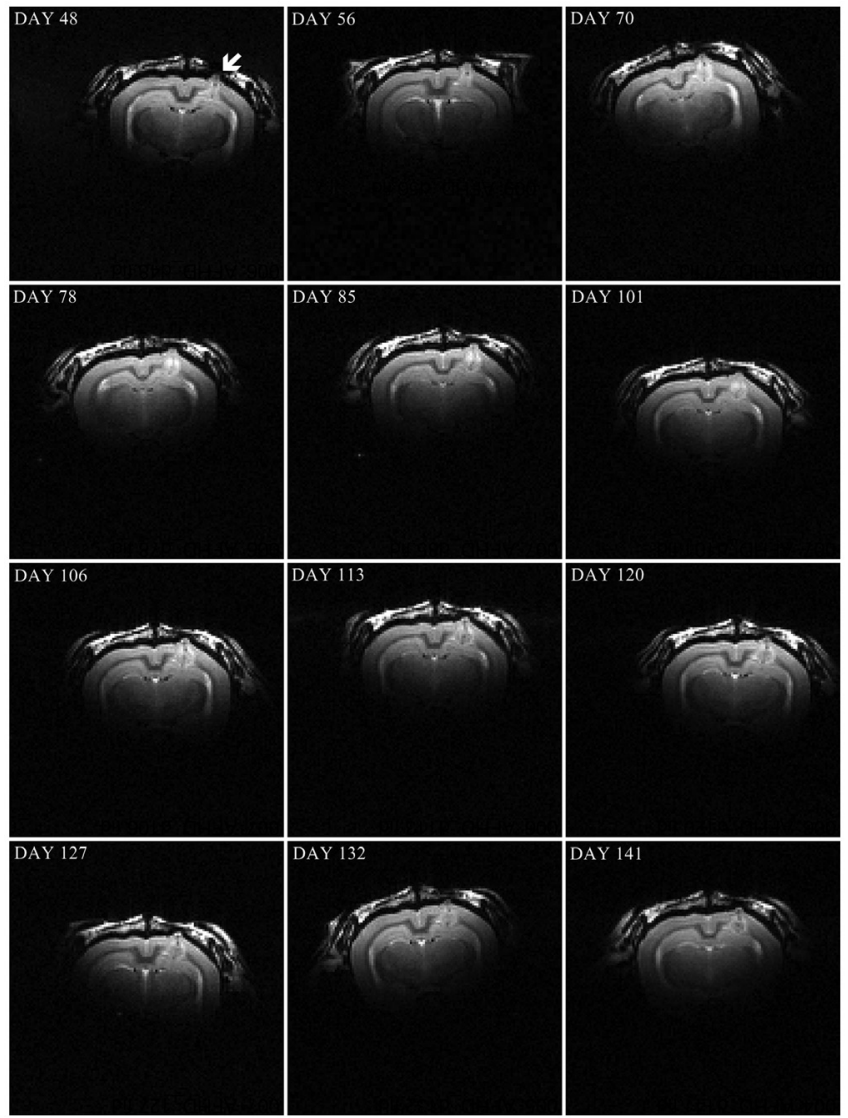

Figure 3. MRI results. MRI data was acquired once every 2 weeks to measure tumor volume. Tumor growth was monitored until day 141, and then the animal was sacrificed.

tumor up to day 141, when the animal was sacrificed (Fig. 4). Notably, the highest tumor volume was measured at day 113, followed by some reduction at the later time points.

Tumor histology and immunostainings. Histological analysis showed that the tumor was similar to a benign low grade ganglioglioma, according to World Health Organization classification of tumors of the central nervous system $(24,25)$. $H \& E$ staining revealed that the tumor recapitulates the emergence of ganglion and glial cells, which are generally characterized as large, rounded with nuclear atypia and multinucleated cells. The presence of microcalcification within the tumor area also marks one of the important features of gangliogliomas (Fig. 5). Similar calcification has been seen in a number of human ganglioglioma cases in cystic lesions (26). The tumor borders were ill-defined, with tumor cells scattered within the surrounding brain tissue, and peritumoral edema was detected. Additionally, within the tumor area, a network-like pattern was observed.

The constitutively active AKT and H-Ras transgenes contain Flag M2 and HA tags, respectively, which act as markers that allow the recognition of proteins. Staining results demonstrated the expression of both proteins in the tumor cells (Fig. 6A and B). In addition, immunostaining of a glial marker in the resultant tumor showed the strong expression of GFAP (Fig. 6C), which is seen in the majority of ganglioglioma subtypes $(26,27)$. p53 protein expression was low in the tumor area. However, we were not able to conclusively

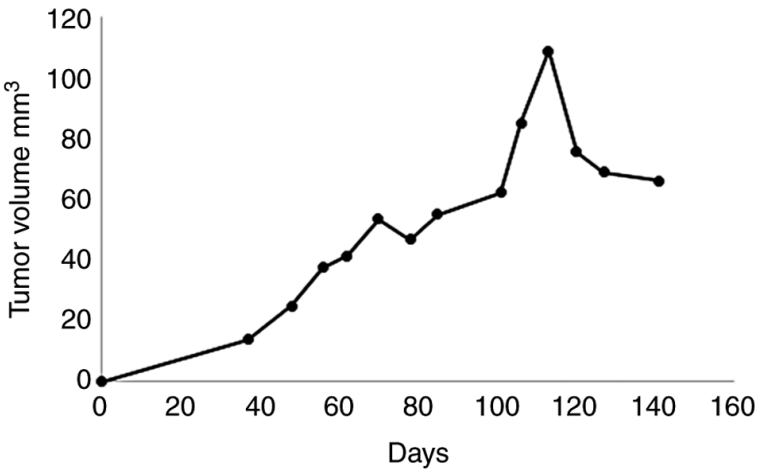

Figure 4. Rabbit ganglioglioma tumor growth. Tumor volumes $\left(\mathrm{mm}^{3}\right)$ were measured from the MRI images.

show that the activity of the tumor suppressor gene may have been blocked by the introduction of the p53 siRNA (Fig. 6D) since the control healthy brain also showed a low expression of the protein. A dense network of small branching capillaries and highly proliferative tumor cells were identified in immunohistochemistry with CD31 and Ki-67 markers, respectively (Fig. 6E and F). Proliferation figures were found in this tumor more frequently compared with a typical grade 1 ganglioglioma but not as frequently as in anaplastic gangliogliomas (28). Furthermore, the slow growth of the tumor during the follow up did not suggest malignant transformation to anaplastic grade 3 ganglioglioma. Therefore, the tumor was defined as a benign grade 2 ganglioglioma. Usually $<5 \%$ of tumor cells express Ki-67 in Grade I gangliogliomas (29).

When investigating the co-localization of AKT and RAS proteins, double staining showed that the tumor composed of heterogeneous cell populations expressed either AKT or $\mathrm{H}$-Ras or both in the same cells (Fig. 6G and H).

H\&E staining showed the presence of tumor cells around the vessels within the tumor area (Fig. 7A and B). Of note, PAS-CD31 dual staining demonstrated the positive expression of PAS lining nearly all CD31-positive channels (Fig. 7C and D). CD31-positive staining was found on the luminal surface of the vessels, whereas a PAS-positive pattern was found on the vessel wall of the intratumoral vessels, which suggested that vascular co-option was primarily in the peritumoral area (30,31). Likewise, vascular co-option occurrence in human astrocytoma has also been reported previously (32). No vasculogenic mimicry was observed.

Re-passaging of the tumor subcutaneously into SCID mice. $\mathrm{H} \& \mathrm{E}$ staining demonstrated the presence of a mixed cell population of reactive ganglion and glial cells in the tumor tissue. Some oligodendroglia cells were also detected, which were characterized by pale nuclei and clear perinuclear area due to inflammation. Additionally, reactive astrocytes showed dark nuclei and atypia (Fig. 8).

\section{Discussion}

In a previous study by Marumoto et al (11) AKT and H-Ras oncogenes were transferred using Cre-LoxP controlled lentivirus vectors into NSCs containing brain regions (subventricular zone or hippocampus) of GFAP-Cre transgenic mice. 

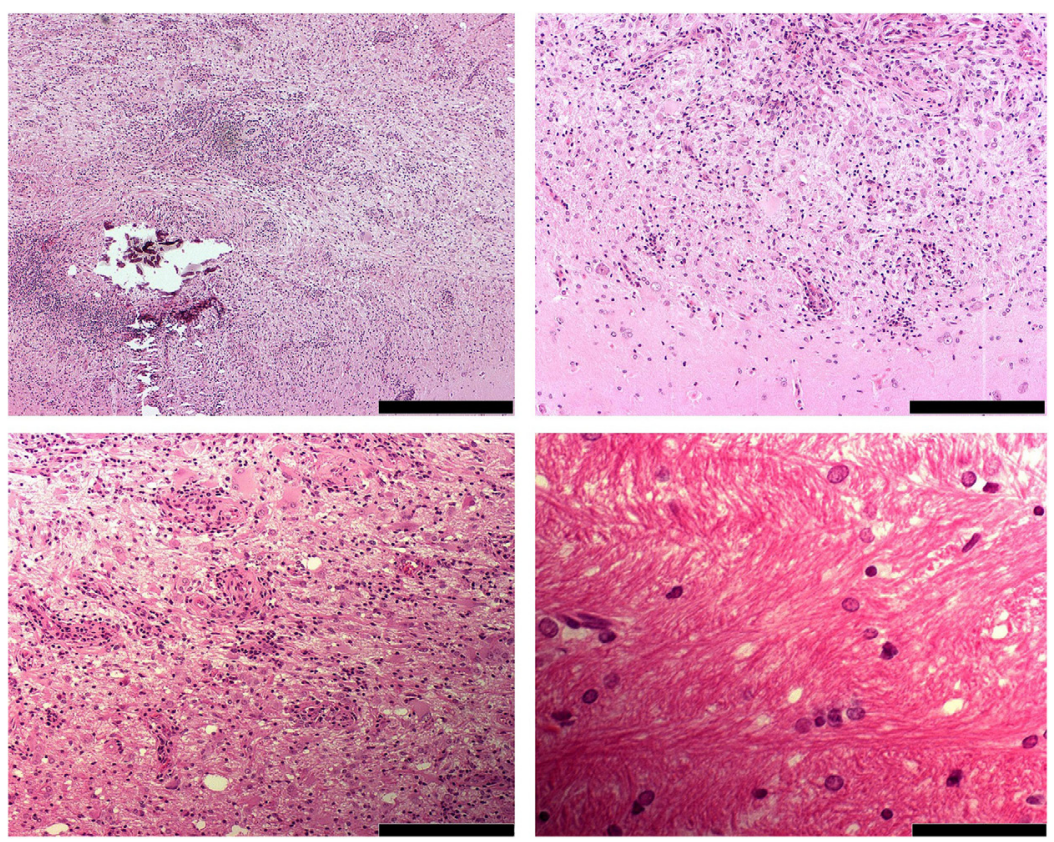

Figure 5. General view of the tumor following hematoxylin and eosin staining. Morphology is similar to ganglioglioma with microcalcification (upper left; magnification, $\mathrm{x} 4$; scale bar, $500 \mu \mathrm{m}$ ). The tumor borders are ill-defined, with tumor cells scattered within the surrounding brain tissue, and peritumoral edema was detected (upper right and lower left; magnification, x10; scale bar, $200 \mu \mathrm{m}$ ). Additionally, an unidentified network-like pattern was observed (lower right; magnification, $\mathrm{x} 40$; scale bar, $50 \mu \mathrm{m})$.

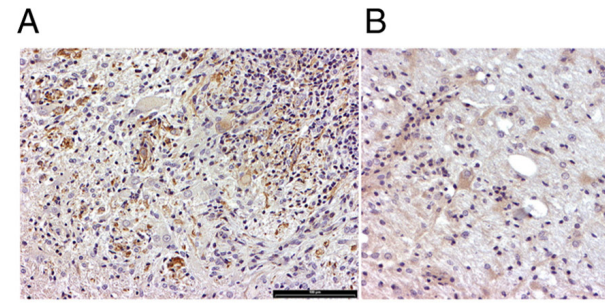

C

$\mathrm{D}$

$\mathrm{E}$

$\mathrm{F}$

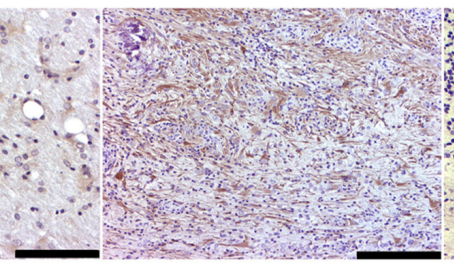

D.........

G

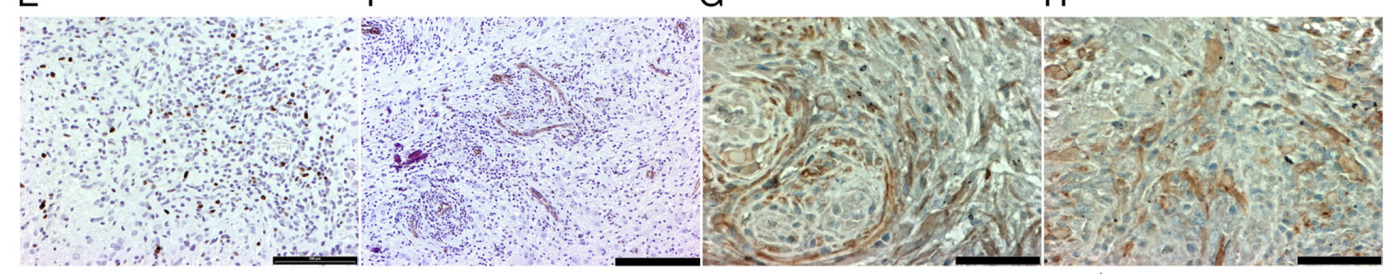

Figure 6. Immunohistochemistry staining. Both (A) AKT and (B) H-Ras transgenes were expressed in the tumor cells (magnification, x20; scale bar, $100 \mu \mathrm{m}$ ). (C) The tumor was characterized by strong GFAP expression (magnification, x10; scale bar, $200 \mu \mathrm{m}$ ). However, (D) low expression of p53 protein was detected (magnification, x20; scale bar, $100 \mu \mathrm{m}$ ). Actively proliferating tumor cells were observed using (E) Ki-67 immunostaining (brown) and hematoxylin counterstain (blue) (magnification, x20; scale bar, $100 \mu \mathrm{m}$ ), and (F) a dense network of small branching capillaries with CD31 (magnification, x10; scale bar, $200 \mu \mathrm{m}$ ). $(\mathrm{G}$ and $\mathrm{H}$ ) Double staining for detecting H-Ras (blue) and AKT (brown) localization/co-localization showed that the tumor was composed of heterogeneous cell populations, which expressed either H-Ras or AKT or both in the same cells (magnification, x40; scale bar, $50 \mu \mathrm{m}$ ).

These mice expressed Cre recombinase in GFAP expressing central nervous system cells, such as mature astrocytes and multipotent NSCs. Gene transfer resulted in the formation of glioblastoma multiforme tumors with frequencies of 10 or $30 \%$ after vector injections into the subventricular zone or hippocampus, respectively. The tumorigenicity was notably enhanced when the mice were also heterozygous for the p53 tumor suppressor gene. In p53 heterozygous mice, vector injections into the subventricular zone and hippocampus yielded glioblastoma formation in 80 and $100 \%$ of the mice, respectively. However, transfer of AKT and H-Ras oncogenes into the cerebral cortex of $\mathrm{p} 53$ heterozygote mice resulted in tumor formation only in 1 out of 15 mice. Most of the histological characteristics of the glioblastoma-like tumors found in the hippocampus or subventricular zone were also present in this cortical tumor (11).

A previous study has reported that NSCs can be isolated and cultured ex vivo followed by gene transfer and implantation in vivo (33). In the present study, newborn NZW rabbit stem cells were isolated and transduced ex vivo with Cre-LoxP controlled lentivirus vectors encoding AKT and H-Ras oncogenes, adenovirus vector carrying Cre recombinase gene 
B
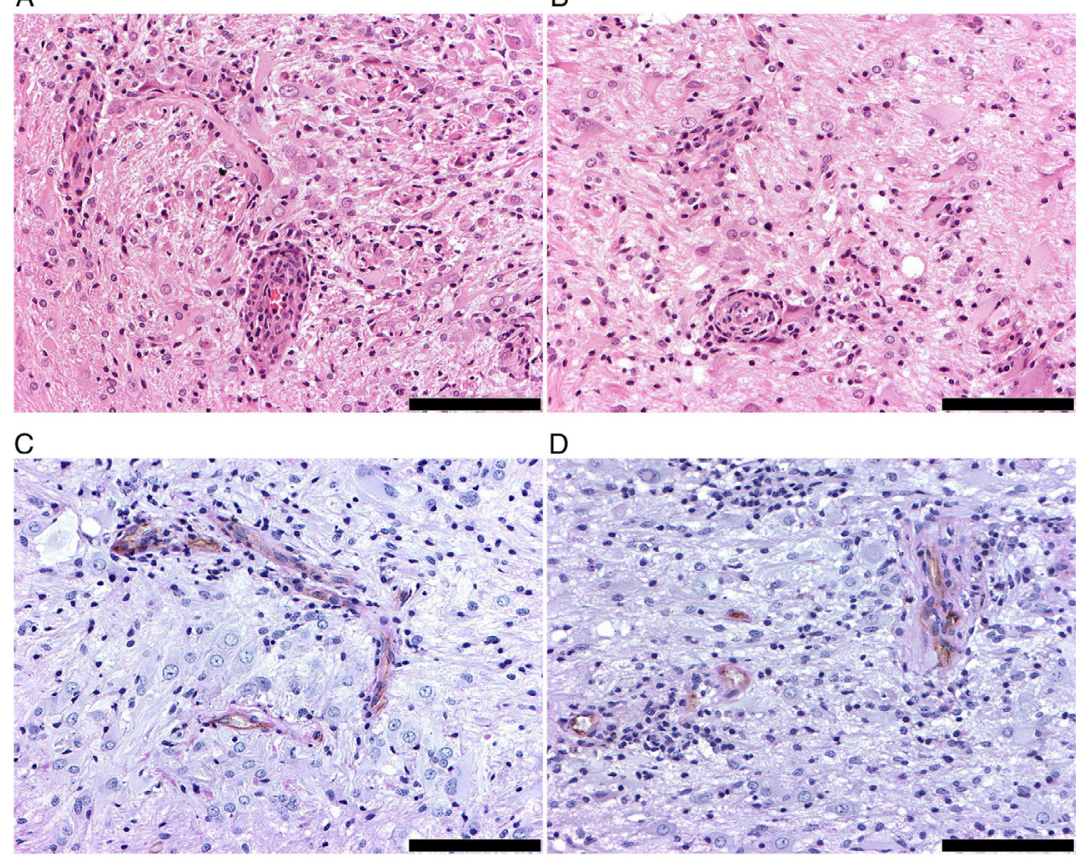

Figure 7. H\&E staining images. (A and B) H\&E results showed the presence of tumor cells around the vessels within the tumor area. (C and D) PAS-CD31 dual immunohistochemistry staining demonstrated PAS-positive staining surrounding nearly all CD31-positive channels. Magnification, x20; scale bar, $100 \mu \mathrm{m}$. H\&E, hematoxylin and eosin; PAS, Periodic acid-Schiff.
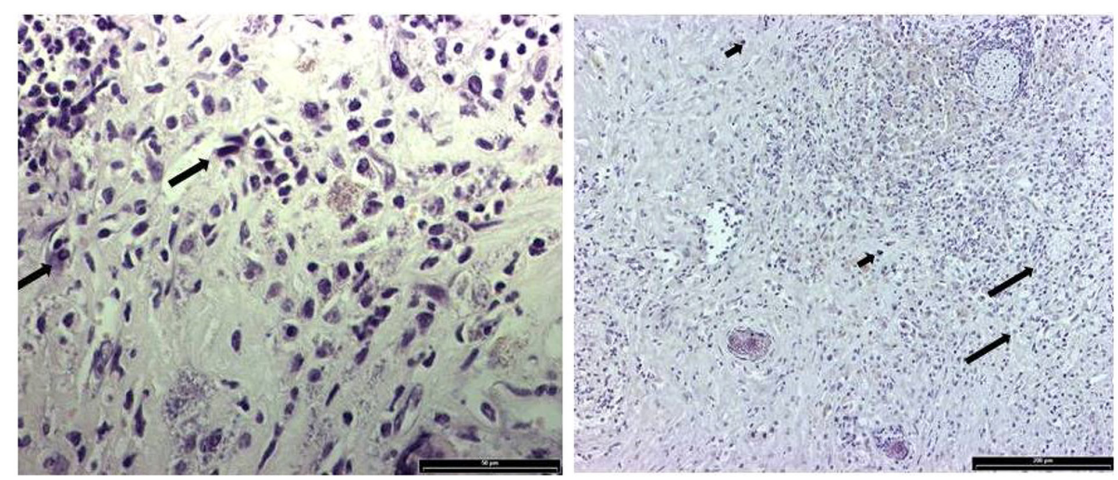

Figure 8. Hematoxylin and eosin staining of the tumor after passaging into severe combined immunodeficient mice. Ganglion cells with nuclear atypia and multinucleated cells were detected (magnification, $\mathrm{x} 40$; scale bar, $50 \mu \mathrm{m}$ ). Long arrows show oligodendrocytes in inflammation, which is characterized by pale nuclei and clear perinuclear area; short arrows show reactive astrocytes with dark nuclei and atypia (magnification, x10; scale bar, $200 \mu \mathrm{m}$ )

and lentivirus vector with p53 siRNA expression cassette. The transduced NSCs were then implanted into the cerebral cortex of NZW rabbits, which is a location that allows surgical resection of tumors (34). Still, with this experimental setting the tumorigenicity was low in the present study. According to histological analysis the tumor represented low grade ganglioglioma.

Ganglioglioma is mostly found in patients younger than 30 years $(30,31)$. In pediatric patients, gangliogliomas represent $\sim 10 \%$ of all primary brain tumors (27). Gangliogliomas are characterized by biphasic histological architecture involving transformed neuronal and glial components (35). Clonality studies suggest that the two different tumor cell populations originate from a single neuroglial precursor cell (36). Gangliogliomas are typically well-differentiated and characterized by slow growth $(37,38)$. Occasionally malignant transformation of the glial component can occur $(37,39,40)$.
It is noteworthy that the use of AKT, H-Ras and p53 siRNA transduced newborn rabbit NSCs for tumor induction resulted in ganglioglioma formation, whereas in the previous study glioblastoma-like tumors arose after injection of the H-Ras and AKT encoding vectors into NSC containing areas in adult p53 heterozygote mouse brains (11).

Several potential reasons for the different pathology of the arising tumor exist. Age of both donor (age-dependent properties of the cells) and recipient animals (growth factors and the tumor microenvironment) may influence tumorigenic properties of NSCs during the malignant transformation process, as well as in resulting histological tumor types. Marumoto et al (11) injected the vectors into the NSC-containing areas of the adult mice, whereas the present study used ex vivo transduction of precultured newborn rabbit NSCs. In newborn mammals, the development of the brains is still ongoing and the NSCs located in the hippocampus and 
subventricular zone play a key role in the process. The NSC population gives rise to radial glial cells (RGCs), which are progenitors for neural and glia cell differentiation that migrate to different areas of the cortical brain and form the neurons and glia cells, including astrocytes and oligodendroglia. Analysis of 326 gangliogliomas by Blümcke and Wiestler (35) revealed that $\sim 80 \%$ of the gangliogliomas expressed CD34-positive staining in immunostaining experiments. CD34 is a stem cell epitope that is not expressed in the normal brain. The majority of these tumors occur in the temporal lobe, which is also the site of the hippocampus, a major NSC-containing region in the brain. Blümcke and Wiestler (35) pointed out that their results supported the idea that ganglioglioma arises from a glioneuronal precursor lesion.

In the present study, the newborn rabbit NSCs were cultured in vitro as neurospheres floating in medium containing EGF and FGF, which have shown to be crucial for maintenance of the NCS phenotype. In absence of these growth factors the NCSs start to differentiate via precursor cells to neurons and glial cells. Without EGF and FGF exposure the cells start to lose their ability to grow as neurospheres (typical feature of the NSCs) and attach to the cell culture plate relatively quickly, within $24 \mathrm{~h}$. The subcortical area is not an anatomical site containing a NSC niche as are the hippocampus or subventricular zone. Instead, the subcortical microenvironment is likely to lack the growth factors and extracellular signals to maintain the NSC phenotype of the implanted cells and rather support the differentiation to neurons and glia cells, likewise the RGCs migrating from the NSC-containing areas of the animal's brain. The present data supported the hypothesis that ganglioglioma arises from a single transformed RGC or NSC committed to differentiate to RGC that then divides and differentiates further, giving rise to two different tumor cell populations, neuronal and glial components of the ganglioglioma.

AKT and H-Ras transgene expression of the tumor was verified with immunostaining. Little is known about the molecular pathogenesis of ganglioglioma, largely because this tumor type is relatively rare. However, there is previous evidence that the PI3K-mTOR signaling pathway, including AKT, is activated in ganglioglioma (41). The present data also supported a previously presented hypothesis, which states that the Ras pathway can play a role in the molecular pathogenesis of ganglioglioma (42).

However, the present study has several limitations. When planning the experiment, it was speculated that the ex vivo culturing of the NSCs may be a process that could potentially change the properties of the cultured cells, especially if the culture is prolonged and continued for multiple passages. Therefore, fluorescence microscopy was selected to count the enhanced (E) GFP or RFP expressing and co-expressing cells for the verification of transgene expression (Fig. 1) After activation with the cre-recombinase, the vectors did not express RFP, but only EGFP in addition to Akt or H-Ras transgene (11). To yield a sufficient number of cultured NSCs for both in vivo implantation and simultaneous flow cytometry analysis would have required significantly longer ex vivo passaging. For the same reason, western blot analysis for confirmation of the p53 knockdown was not carried out with the ex vivo cultured and transduced rabbit NSCs of the same batch that was used for the in vivo experiment. Therefore, one of the major limitations of this study was that we were unable to indisputably show p53 expression suppression by p53 siRNA. The immunostaining experiment showed very low p53 expression within the tumor area; however, the finding was similar in the surrounding normal brain tissue. In a previous study by Marumoto et al (11) with AKT and H-Ras transgene-induced tumors, the proportion of Ki-67-positive tumor cells increased from $\sim 1$ to $5.5 \%$ when p53 heterozygous mice were used instead of mice who expressed p53 normally. In the present study, a high number of Ki-67 expressing cells were detected, which indicated tumor cell proliferation and the reduced activity or inactivation of p53. Still, the MRI follow-up indicated a reduction in tumor size post-day 120 . The reason for this remains to be elucidated. However, gradual weakening of siRNA-mediated p53 blockade during the relatively long follow-up cannot be excluded. Due to these limitations of the experimental design, future studies, especially on the role of $\mathrm{p} 53$ in the pathogenesis of ganglioglioma, are warranted. Since the aim of the present study was to develop a new rabbit model for malignant glioma, the study was not repeated to avoid using animals for such experiments that were unlikely to serve this purpose. The lack of data supporting the reproducibility of these findings also remains a major limitation of the present study.

A few case reports with molecular genetic analysis indicate that in rare occasions malignant transformation of ganglioglioma to high grade glioma can occur. These reports suggest the role of p53 inactivation in this process $(39,43)$. In the present study, the rabbit ganglioglioma tumor showed a relatively high number of Ki-67-positive cells, which suggested the malignant transformation to anaplastic ganglioglioma.

Previous results demonstrated that injection of AKT and H-Ras encoding vectors into the mouse cerebral cortex resulted in tumor formation, but tumorigenicity was low (11). Lower tumorigenicity and different histological characteristics of the resulting tumor compared with a previous study in mice (11) could be the result of several factors. It seems likely that this could be related to the absence or low number of NSCs in the cerebral cortex compared with the hippocampus or subventricular zone, the known areas that contain NSCs in the brain. Instead of direct vector injection, ex vivo transduced NSCs (isolated from newborn NZW rabbit hippocampus and subventricular zone) were injected into the cerebral cortex of NZW rabbits into a location favorable for tumor resection surgery. The tumorigenicity remained low despite the use of NSCs cultured ex vivo. This may be related to injection site-dependent factors, such as growth factors, which are absent in the cortical brain tissue compared with the hippocampus. Microenvironment and growth factors may be important in the early phase of tumor formation. The properties of NSCs may have changed, perhaps due to the differentiation to RGCs before the start of the transgene expression. Different cell types may present variation in the sensitivity to oncogenic stress, such as lentivirus vector-driven overexpression of AKT and H-Ras genes. Both AKT/mTOR and FGF/Ras/MAPK/ETV signaling pathways are known to play roles in the natural process of RGC differentiation from NSCs (44), and this may explain the relative resistance of NSCs for the tumorigenic effects of AKT and H-Ras transgenes in the present study. When the cell is committed to a differentiation process involving activation of 
these signaling pathways, it may have turned on its inherent protective mechanisms for the adverse oncogenic effects of activation of these particular signaling pathways. There is evidence that cells have inherent mechanisms to protect themselves against the oncogenic effects of the signaling pathways needed for their normal actions in tissue regeneration (45-47). In a previous study by Marumoto et al (11), the NCSs were transduced with oncogenes in their natural environment, the stem cell niche located in the hippocampus or subventricular zone, without simultaneously taking them to an environment less favorable for maintaining the stemness of the cells. We hypothesize that the already ongoing process of differentiation to RGCs may protect the NSCs from developing into glioblastoma multiforme, and instead lead to the formation of a far more benign ganglioglioma or in most cases completely prevent tumor formation.

The animal studies were conducted between June 2008 and August 2010. At that time, we were unable to understand the findings, i.e. the emergence of a ganglioglioma tumor, instead of the expected glioblastoma. However, the recently published findings on the molecular mechanisms of the NSC differentiation processes have now enabled us to interpret the results of the present study $(41,42,44)$.

In conclusion, lentiviral vectors provide a tool for the manipulation of oncogenic molecular pathways to induce tumor growth in the rabbit brain. Thereby, in addition to their benefits for the development of rodent cancer models and investigating the roles of different oncogenes, they could be useful for establishing larger animal models, such as resectable malignant glioma in rabbits. Moreover, it is hoped that these findings on ganglioglioma formation will stimulate further research on NSC differentiation, interferences in the differentiation process and pathogenesis of ganglioglioma, a rare tumor type associated with drug-resistant focal epilepsy.

\section{Acknowledgements}

The authors would like to thank Dr Inder Verma (Salk Institute, USA) for providing the lentiviral vectors.

\section{Funding}

This study was supported by grants from Finnish Academy (grant no. 124222) and Sigrid Juselius Foundation.

\section{Availability of data and materials}

All data generated or analyzed during this study are included in this published article.

\section{Authors' contributions}

FA was involved in acquisition of the data, especially the in vitro and animal studies, MRI and histologic data, as well as analysis and interpretation of the data. AH was involved in study design, acquisition of the data, especially the in vitro and animal studies, MRI and histologic data, and analysis and interpretation of the data. AP was involved in acquisition of the data, especially the in vitro and animal studies and MRI data, and analysis and interpretation of the data. VO was involved in acquisition of the data, especially the animal studies and histology, as well as the analysis and interpretation of the data. JR was involved in the study design and interpretation of the histology of the tumor. AI was involved in the study design, development of the operative technique and instrumentation suitable for rabbits together with $\mathrm{AH}$, and the interpretation of the data. JN was involved in study design, design of the MRI sequences and instructions on the MRI imaging, and interpretation of the data. PT was involved in study design, design of the MRI sequences and instructions on the MRI imaging, and interpretation of the data. TL was involved in study design, design of the MRI sequences and instructions on the MRI imaging, and interpretation of the data. VK was involved in study design, stem cell isolations together with AH, FA and AP, and interpretation of the data. JK was involved in study design and interpretation of the data. SYH was involved in study design and interpretation of the data. FA, AH and VO confirm the authenticity of the raw data. All authors read and approved the final manuscript.

\section{Ethics approval and consent to participate}

The study was carried out according to Finnish National and European Union legislation and guidelines. The permission for animal studies was acquired from the Project Authorization Board of the Regional State Administrative Agency.

\section{Patient consent for publication}

Not applicable.

\section{Competing interests}

The authors declare that they have no competing interests.

\section{References}

1. Lönn S, Klaeboe L, Hall P, Mathiesen T, Auvinen A, Christensen HC, Johansen C, Salminen T, Tynes T and Feychting M: Incidence trends of adult primary intracerebral tumors in four Nordic countries. Int J Cancer 108: 450-455, 2004.

2. Houben MP, Aben KK, Teepen JL, Schouten-Van Meeteren AY, Tijssen CC, Van Duijn CM and Coebergh JW: Stable incidence of childhood and adult glioma in the Netherlands, 1989-2003. Acta Oncol 45: 272-279, 2006.

3. Merchant TE, Pollack IF and Loeffler JS: Brain tumors across the age spectrum: Biology, therapy, and late effects. Semin Radiat Oncol 20: 58-66, 2010.

4. Candolfi M, Curtin JF, Nichols WS, Muhammad AG, King GD, Pluhar GE, McNiel EA, Ohlfest JR, Freese AB, Moore PF, et al: Intracranial glioblastoma models in preclinical neuro-oncology: Neuropathological characterization and tumor progression. J Neurooncol 85: 133-148, 2007.

5. Huse JT and Holland EC: Genetically engineered mouse models of brain cancer and the promise of preclinical testing. Brain Pathol 19: 132-143, 2009.

6. Laerum OD and Rajewsky MF: Neoplastic transformation of fetal rat brain cells in culture after exposure to ethylnitrosourea in vivo. J Natl Cancer Inst 55: 1177-1187, 1975.

7. Laerum OD, Rajewsky MF, Schachner M, Stavrou D, Haglid KG and Haugen A: Phenotypic properties of neoplastic cell lines developed from fetal rat brain cells in culture after exposure to ethylnitrosourea in vivo. Z Krebsforsch Klin Onkol Cancer Res Clin Oncol 89: 273-295, 1977.

8. Sampson JH, Ashley DM, Archer GE, Fuchs HE, Dranoff G, Hale LP and Bigner DD: Characterization of a spontaneous murine astrocytoma and abrogation of its tumorigenicity by cytokine secretion. Neurosurgery 41: 1365-1373, 1997. 
9. Serano RD, Pegram CN and Bigner DD: Tumorigenic cell culture lines from a spontaneous VM/Dk murine astrocytoma (SMA). Acta Neuropathol 51: 53-64, 1980

10. de Vries NA, Bruggeman SW, Hulsman D, de Vries HI, Zevenhoven J, Buckle T, Hamans BC, Leenders WP, Beijnen JH, van Lohuizen M, et al: Rapid and robust transgenic high-grade glioma mouse models for therapy intervention studies. Clin Cancer Res 16: 3431-3441, 2010.

11. Marumoto T, Tashiro A, Friedmann-Morvinski D, Scadeng M, Soda Y, Gage FH and Verma IM: Development of a novel mouse glioma model using lentiviral vectors. Nat Med 15: 110-116, 2009.

12. Kanu OO, Hughes B, Di C, Lin N, Fu J, Bigner DD, Yan H and Adamson C: Glioblastoma multiforme oncogenomics and signaling pathways. Clin Med Oncol 2009: 39-52, 2009.

13. Barker FG, Chang SM, Larson DA, Sneed PK, Wara WM, Wilson CB and Prados MD: Age and radiation response in glioblastoma multiforme. Neurosurgery 49: 1288-1298, 2001.

14. Ng WH, Wan GQ and Too HP: Higher glioblastoma tumour burden reduces efficacy of chemotherapeutic agents: In vitro evidence. J Clin Neurosci 14: 261-266, 2007.

15. Stewart LA: Chemotherapy in adult high-grade glioma: A systematic review and meta-analysis of individual patient data from 12 randomised trials. Lancet 359: 1011-1018, 2002.

16. Katayama N, Sugimoto K, Okada T, Ueha T, Sakai Y, Akiyoshi H, Mie K, Ueshima E, Sofue K, Koide Y, et al: Intra-arterially infused carbon dioxide-saturated solution for sensitizing the anticancer effect of cisplatin in a rabbit VX2 liver tumor model. Int J Oncol 51: 695-701, 2017.

17. Flecknell P: Laboratory animal anaesthesia, Third edition. Acad Press, 2009.

18. Clarke DL, Johansson CB, Wilbertz J, Veress B, Nilsson E, Karlström H, Lendahl U and Frisén J: Generalized potential of adult neural stem cells. Science 288: 1660-1663, 2000.

19. Castrén M, Tervonen T, Kärkkäinen V, Heinonen S, Castrén E, Larsson K, Bakker CE, Oostra BA and Akerman K: Altered differentiation of neural stem cells in fragile $\mathrm{X}$ syndrome. Proc Natl Acad Sci USA 102: 17834-17839, 2005.

20. Ikawa M, Tanaka N, Kao WW and Verma IM: Generation of transgenic mice using lentiviral vectors: A novel preclinical assessment of lentiviral vectors for gene therapy. Mol Ther 8: 666-673, 2003.

21. Follenzi A and Naldini L: HIV-based vectors. Preparation and use. Methods Mol Med 69: 259-274, 2002.

22. Leppänen P, Kholová I, Mähönen AJ, Airenne K, Koota S, Mansukoski H, Närväinen J, Wirzenius M, Alhonen L, Jänne J, et al: Short and long-term effects of hVEGF-A(165) in Cre-activated transgenic mice. PLoS One 1: e13, 2006.

23. Close B, Banister K, Baumans V, Bernoth EM, Bromage N, Bunyan J, Erhardt W, Flecknell P, Gregory N, Hackbarth H, et al: Recommendations for euthanasia of experimental animals: Part 1. DGXI of the European Commission. Lab Anim 30: 293-316, 1996.

24. Louis DN, Ohgaki H, Wiestler OD, Cavenee WK, Burger PC, Jouvet A, Scheithauer BW and Kleihues P: The 2007 WHO classification of tumours of the central nervous system. Acta Neuropathol 114: 97-109, 2007.

25. Louis DN, Perry A, Reifenberger G, von Deimling A, Figarella-Branger D, Cavenee WK, Ohgaki H, Wiestler OD, Kleihues P and Ellison DW: The 2016 World Health Organization Classification of Tumors of the Central Nervous System: A summary. Acta Neuropathol 131: 803-820, 2016.

26. Zhang D, Henning TD, Zou LG, Hu LB, Wen L, Feng XY, Dai SH, Wang WX, Sun QR and Zhang ZG: Intracranial ganglioglioma: Clinicopathological and MRI findings in 16 patients. Clin Radiol 63: 80-91, 2008.

27. Karremann M, Pietsch T, Janssen G, Kramm CM and Wolff JE: Anaplastic ganglioglioma in children. J Neurooncol 92: 157-163, 2009.

28. Rogojan L and Olinici CD: Ganglioglioma with glioblastoma component. Rom J Morphol Embryol 49: 403-406, 2008.
29. Rousseau A, Kujas M, Bergemer-Fouquet AM, Effenterre R and Hauw JJ: Survivin expression in ganglioglioma. J Neurooncol 77: 153-159, 2006.

30. Miller DC, Lang FF and Epstein FJ: Central nervous system gangliogliomas. Part 1: Pathology. J Neurosurg 79: 859-866, 1993.

31. Mpairamidis E, Alexiou GA, Stefanaki K, Sfakianos G and Prodromou N: Brainstem ganglioglioma. J Child Neurol 23: 1481-1483, 2008.

32. Yue WY and Chen ZP: Does vasculogenic mimicry exist in astrocytoma? J Histochem Cytochem 53: 997-1002, 2005.

33. Jandial R, Singec I, Ames CP and Snyder EY: Genetic modification of neural stem cells. Mol Ther 16: 450-457, 2008.

34. Ahmad F, Pacholska A, Tuppurainen V, Ylä-Herttuala S and Hyvärinen A: Resectable VX-2 rabbit brain tumor model for development of intraoperative local administration of drugs. Acta Neurochir (Wien) 153: 1979-1981, 2011.

35. Blümcke I and Wiestler OD: Gangliogliomas: An intriguing tumor entity associated with focal epilepsies. J Neuropathol Exp Neurol 61: 575-584, 2002.

36. Zhu JJ, Leon SP, Folkerth RD, Guo SZ, Wu JK and Black PM: Evidence for clonal origin of neoplastic neuronal and glial cells in gangliogliomas. Am J Pathol 151: 565-571, 1997.

37. Im SH, Chung CK, Cho BK, Wang KC, Yu IK, Song IC, Cheon GJ, Lee DS, Kim NR and Chi JG: Intracranial ganglioglioma: Preoperative characteristics and oncologic outcome after surgery. J Neurooncol 59: 173-183, 2002.

38. Luyken C, Blümcke I, Fimmers R, Urbach H, Wiestler OD and Schramm J: Supratentorial gangliogliomas: Histopathologic grading and tumor recurrence in 184 patients with a median follow-up of 8 years. Cancer 101: 146-155, 2004.

39. Pandita A, Balasubramaniam A, Perrin R, Shannon P and Guha A: Malignant and benign ganglioglioma: A pathological and molecular study1. Neuro Oncol 9: 124-134, 2007.

40. Zentner J, Wolf HK, Ostertun B, Hufnagel A, Campos MG, Solymosi L and Schramm J: Gangliogliomas: Clinical, radiological, and histopathological findings in 51 patients. J Neurol Neurosurg Psychiatry 57: 1497-1502, 1994.

41. Boer K, Troost D, Timmermans W, van Rijen PC, Spliet WG and Aronica E: Pi3K-mTOR signaling and AMOG expression in epilepsy-associated glioneuronal tumors. Brain Pathol 20: 234-244, 2010.

42. Pekmezci M, Villanueva-Meyer JE, Goode B, Van Ziffle J, Onodera C, Grenert JP, Bastian BC, Chamyan G, Maher OM, Khatib Z, et al: The genetic landscape of ganglioglioma. Acta Neuropathol Commun 6: 47, 2018.

43. Kim NR, Wang KC, Bang JS, Choe G, Park Y, Kim SK, Cho BK and Chi JG: Glioblastomatous transformation of ganglioglioma: Case report with reference to molecular genetic and flow cytometric analysis. Pathol Int 53: 874-882, 2003.

44. Penisson M, Ladewig J, Belvindrah R and Francis F: Genes and mechanisms involved in the generation and amplification of basal radial glial cells. Front Cell Neurosci 13: 381, 2019.

45. Wang H, Lafdil F, Wang L, Park O, Yin S, Niu J, Miller AM, Sun Z and Gao B: Hepatoprotective versus oncogenic functions of STAT3 in liver tumorigenesis. Am J Pathol 179: 714-724, 2011.

46. Guadagno E, Vitiello M, Francesca P, Calì G, Caponnetto F, Cesselli D, Camorani S, Borrelli G, Califano M, Cappabianca P, et al: PATZ1 is a new prognostic marker of glioblastoma associated with the stem-like phenotype and enriched in the proneural subtype. Oncotarget 8: 59282-59300, 2017.

47. Vitiello M, Palma G, Monaco M, Bello AM, Camorani S, Francesca P, Rea D, Barbieri A, Chiappetta G, Vita G, et al: Dual oncogenic/anti-oncogenic role of PATZ1 in FRTL5 rat thyroid cells transformed by the Ha-Ras ${ }^{\mathrm{v} 2}$ oncogene. Genes (Basel) 10: 127, 2019.

This work is licensed under a Creative Commons Attribution-NonCommercial-NoDerivatives 4.0 International (CC BY-NC-ND 4.0) License. 